\title{
Uncoupled modes and all-angle negative refraction in walled honeycomb photonic crystals
}

\author{
Ken-Ming Lin and G. Y. Guo* \\ Department of Physics and Center for Theoretical Sciences, National Taiwan University, Taipei 106, Taiwan \\ *Corresponding author: gyguo@phys.ntu.edu.tw
}

Received May 14, 2008; accepted July 23, 2008;

posted August 11, 2008 (Doc. ID 96148); published September 18, 2008

\begin{abstract}
Left-handed materials have superlensing effects that enable them to surmount the optical diffraction limit. A photonic crystal is able to mimic some properties of all-angle left-landed materials. In this study, the all-angle negative refraction criteria of photonic crystals are evaluated. The MIT Photonic-Bands program is employed to calculate the band structure of walled honeycomb photonic crystals, and the finite-difference time-domain method is used to provide a snapshot of the electric field distribution inside and outside the honeycomb photonic crystals. The results indicate that the all-angle negative refraction phenomena of the honeycomb photonic crystals are correlated with the orientation of the photonic crystals. Furthermore, the role of the uncoupled modes varies based on their orientation to the all-angle negative refraction photonic crystals, in one case assisting negative refraction and in the other case preventing it. The results suggest that symmetric properties should not be ignored when considering the negative refraction of photonic crystals. (C) 2008 Optical Society of America

OCIS codes: $160.4760,160.5298,220.3620,260.2065,350.3618$.
\end{abstract}

\section{INTRODUCTION}

Photonic crystals are made by periodically modulated refraction index materials [1-5]. They may bend light in an abnormal direction. One interesting behavior exhibited by photonic crystals is negative refraction. Veselogo proved that several abnormal phenomena accompany lefthanded materials (LHM) composed of simultaneously negative dielectric permittivity $\varepsilon(r)$ and negative magnetic permeability $\mu(r)$ [6]. For example, LHM exhibit reverse Doppler effects. Negative refraction occurs when a light beam enters from normal materials to LHM. A source point can pass through the LHM plate and focus into another point. The phase velocity of the light wave propagating inside this material is opposite to the group velocity. And $\mathbf{E}, \mathbf{H}$, and $\mathbf{k}$ form a left-handed set.

Pendry reported that LHM have a superlensing effect that overcomes diffraction limits [7]. Using a LHM lens, image sharpness is not limited by the wavelength of light. Nevertheless, there is no material of this kind in nature. It means LHM must be artificial, either metamaterial or photonic crystal. Metamaterial is made from a periodic array of conductive split ring resonators and metallic wires [8]. Shelby et al. observed that the metamaterial's effective index of refraction in a microwave frequency band is negative [9]. On the other hand, several researchers have used photonic crystals to reproduce some properties of LHM. Luo et al. found that reverse Doppler effects inside photonic crystals have similar behaviors in LHM [10]. Notomi proved that negative refraction and negative group velocity exist in some two-dimensional photonic crystals [11]. The effective refractive index $n_{\text {eff }}$ of a photonic crystal is determined by its band structure. In these photonic crystals the permeability is equal to one, and the periodically modulated permittivity is positive.
Luo et al. [12] and Parimi et al. [13] have reported that there is an all-angle negative refraction effect in photonic crystals. Its frequency of negative refraction is smaller than at the top of the hole band but larger than at the intersection of the light line and the band structure. A source point can pass through the photonic crystal slab and focus into another point, and it is impossible to produce this phenomenon using a conventional planar lens. The size of the image suggests that there are superlensing effects. The subwavelength resolution is also achieved for two point images from two point sources whose separation is smaller than a wavelength $[14,15]$. Few authors have noted that the focusing of the square lattice is not caused by the superlensing effects, but rather by selfguiding and complex near-field wave scattering effects [16-19]. Foteinopoulou et al. distinguished the negative refraction from left-handed behavior in the twodimensional photonic crystals [20]. They pointed out that the negative refraction materials do not ensure lefthanded behavior. Only materials that have a phase velocity which are antiparallel to the group velocity of the photonic crystals are called LHM.

To construct LHM using photonic crystals the crystals need to possess certain special characteristics [11,12,20,21]. First, they should have equal frequency contours that are nearly isotropic in a band region where the phase velocity and the group velocity are antiparallel. Since the equal frequency contours are almost isotropic, in principle negative refraction can occur at any incident angle of the external wave. To accomplish this, triangular, hexagonal, or kagomé lattices of photonic crystals have been previously chosen and the $\Gamma$ point has been selected as the center of the circular equal frequency contour [22]. Second, the radius of the equal frequency contour needs 
to be larger than that of air. Third, any higher-order Bragg reflection for any incident direction should be absent. Finally, a single beam propagation should be guaranteed within the photonic crystals.

However, Robertson et al. [23] and Sakoda [4,24,25] have shown that an external incident plane-wave radiation cannot excite some modes when symmetry is taken into consideration. These modes, which cannot be excited by external incident waves, are called uncoupled modes. While many studies have been done on the all-angle negative refraction in photonic crystals, little information is available on the uncoupled modes in the all-angle negative refraction photonic crystals [26]. The purpose of the present paper is to determine whether the criteria for the all-angle negative refraction mentioned above is sufficient, and whether the uncoupled modes can be excited in all-angle negative refraction photonic crystals. The results of this study may lead to a better understanding of the roles of uncoupled modes in all-angle negative refraction photonic crystals. This information could also be useful in developing negative refraction devices.

\section{COMPUTATIONAL METHOD AND MATERIALS}

In this paper, the dielectric cylinders (along the $z$ direction) of two-dimensional honeycomb photonic crystals (periodic arrayed in the $x-y$ plane) (Fig. 1), as in [27,28], were studied and their transverse-magnetic (TM) mode band where negative refraction occurred was considered (the electric field is parallel to the rods). The waves, which had an angle of incidence oblique from the $\Gamma-K$ direction and normal to the $\Gamma-K$ direction of the honeycomb photonic crystal, were simulated and compared (Fig. 1).

The band structure and the equal frequency contours were calculated using the plane-wave expansion method [29-31], as implemented in the MIT Photonic-Bands (MPB) package [32]. Fully vectorial eigenmodes of Maxwell's equations with periodic boundary conditions were computed using preconditioned conjugate-gradient minimization of the block Rayleigh quotient on a plane-wave basis.

To compute the propagation of electromagnetic waves in photonic crystals we developed a program that uses the finite-difference time-domain (FDTD) method [33-36]. The FDTD program is based on the differential form of Maxwell's equation. The two curl equations of Maxwell's equation are transformed into two difference equations by taking the central difference approximations, in which the continuous variables for position and time become discrete variables. The electric field of each position point of each time step can be calculated from the electric field of the previous time step at the same position point and the magnetic field of the previous half-time-step at the neighboring points. The magnetic field is also determined by the magnetic field of the previous time step and the surrounding electric field of the previous half-time-step. Near the calculation boundaries, the perfect matched layer (PML) is used to absorb the electromagnetic waves without any reflection [34]. The amplitude of electromagnetic waves is decreased by the absorption within the PML. At the calculation boundaries the electric field is directly given a value of zero. In the beginning the electric field is assumed to be zero. Using these initial conditions, boundary conditions, and the processes of iteration, the time evolution of the electromagnetic waves can be derived in the calculation space. The electric and magnetic fields of discrete mesh points are stored in two-dimensional matrices for the current time step. The size of the matrices are determined by the physical size of computer memory. To increase the spatial resolution and reduce the execution time of the FDTD program, we also used the messagepassing interface (MPI) standard to parallelize the FDTD program [37-39]. The parallel algorithm of the MPI FDTD program uses a domain decomposition, and the data is divided into pieces among the multiple processors used. Because the field updated at a mesh point requires the field values of the surrounding points, transmission of the field values is required by MPI functions between do-

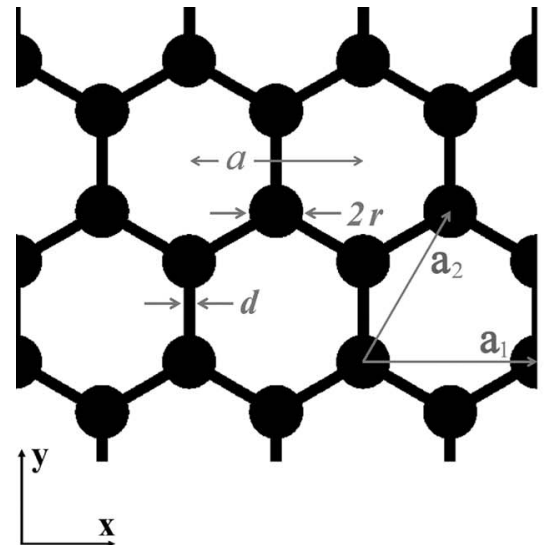

(a)

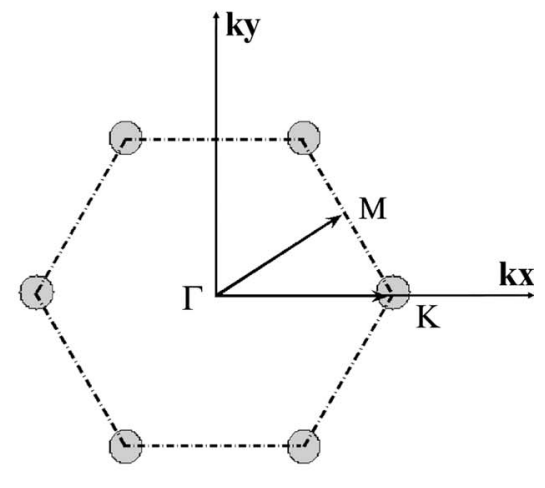

(b)

Fig. 1. (a) Two-dimensional honeycomb photonic crystals: $r$ is the radius of the cylinders, $d$ is the thickness of the connecting walls, and $a$ is the lattice constant of the honeycomb photonic crystals. $\mathbf{a}_{1}$ and $\mathbf{a}_{2}$ are the bases of the primitive unit cell of the honeycomb. (b) First Brillouin zone of the honeycomb photonic crystals. The irreducible Brillouin zone is enclosed by the lines joining the points $\Gamma, K$, and $M$. 
main boundaries. Adding processors reduces the computation time but increases the communication time. The communication overhead between the processors slows the speed-up. For any fixed problem size, an optimum number of processors exist in minimizing overall parallel execution time.

The systems considered are two-dimensional honeycomb photonic crystals made of arrays of parallel dielectric cylinders and connecting dielectric walls placed in the vacuum (Fig. 1) [27,28]. The primitive unit cell of the honeycomb has the triangular basis $\mathbf{a}_{1}=a(1,0)$ and $\mathbf{a}_{2}$ $=a\left(1 / 2,3^{1 / 2} / 2\right)$ with two cylinders per primitive cell. The lattice constant $a$ is the distance between the centers of the two hexagons. The material from the two-dimensional honeycomb photonic crystals is regarded as linear, isotropic, lossless, nondispersive, and nonmagnetic. Both the dielectric constants of the cylinders and the connecting walls are $\varepsilon / \varepsilon_{0}=13.0$. In the two-dimensional honeycomb photonic crystals there are three geometric parameters: the lattice constant $a$, the radius of the cylinders $r / a$ $=0.155$, and the thickness of the connecting walls $d / a$ $=0.065$. For convenience, all lengths are scaled using the lattice constant. The bandgaps occur in the isolated high dielectric structures for the TM modes. However, the bandgaps take place in the connected structures for the transverse-electric (TE) modes [3]. Combining the isolated and connected structures, the full bandgaps of the two-dimensional photonic crystals may be obtained. The largest full bandgap of the two-dimensional photonic crystals was found for $r / a=0.155$ and $d / a=0.035$ [27].

\section{RESULTS AND DISCUSSION}

Figure 2 displays the band structure of the twodimensional honeycomb photonic crystals for TM mode with $\varepsilon / \varepsilon_{0}=13, r / a=0.155$, and $d / a=0.065$. Since the bandgap occurs at the zone boundaries of irreducible Brillouin zone, the frequencies were plotted along lines in the irreducible Brillouin zone joining the points $\Gamma=(0,0), K$ $=(2 / 3,0) \times 2 \pi / a$, and $M=\left(1 / 2,1 /\left(2 \times 3^{1 / 2}\right)\right) \times 2 \pi / a$. There

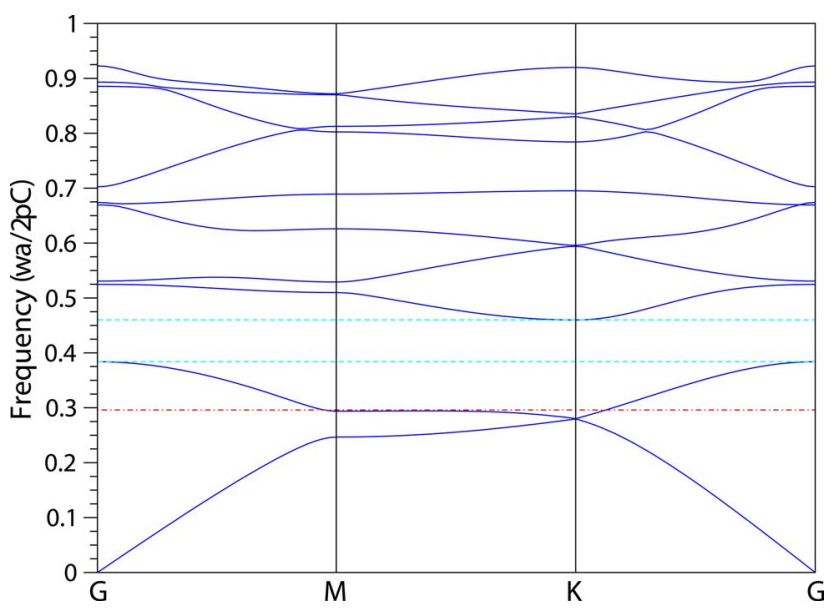

Fig. 2. (Color online) Band structure of the two-dimensional honeycomb photonic crystals for TM mode with $\varepsilon / \varepsilon_{0}=13, r / a$ $=0.155$, and $d / a=0.065$. The zone between two horizontal dashed lines corresponds to the band gap region. The all-angle negative refraction frequency $f=0.295 \times 2 \pi C / a$ in this study is indicated as the dashed-dotted line. was a bandgap between $0.384 \times 2 \pi C / a$ and 0.460 $\times 2 \pi C / a$, and a hole band with a negative effective mass $\partial^{2} \omega / \partial k_{i} \partial k_{j}$ was found at the $\Gamma$ point of the second band. The overall trend of the first and second band is similar to the first and second bands of triangular photonic crystals.

A possibility for the all-angle negative refraction is the second band of the TM mode whose equal frequency contours are shown in Fig. 3. There are several criteria for choosing the frequency of the incident wave from air to reveal the all-angle negative refraction. (i) The convex equal frequency contours of negative refraction are selected. Near the top of the second band of the $\Gamma$ point, the direction of group velocity $\mathbf{V}_{g}=\nabla_{\mathbf{k}} \omega$ is normal to the equal frequency contour and inward to the $\Gamma$ point. (ii) The radius of the equal frequency contour of the negative refraction frequency is larger than the magnitude of incoming wave vectors from all directions at such a frequency to insure that all incoming waves at the definite frequency are excited in the photonic crystals. (iii) The selected frequencies are smaller than $0.5 \times 2 \pi C / a$ to avoid scattering effects. (iv) Choosing the contour whose shape closely approximates a circle simulates an isotropic medium.

If a light beam is launched into this honeycomb photonic crystal with the wave vector $\mathbf{k}_{\text {air }}$ across the $\Gamma-K$ direction at the frequency $f=0.295 \times 2 \pi C / a$, a negative refraction effect is expected (as is the case in Fig. 3). Since there is periodic variance of the dielectric constant between the honeycomb photonic crystal and the air in the direction of the interface, there is the tangential wave vectors's conservation between the incident and refraction waves with allowances for the difference of a multiple of $(2 / 3,0) \times 2 \pi / a$. The Bloch wave function for a given band index $n$ is identical for two values of $\mathbf{k}$ differing by a reciprocal lattice vector $\psi_{n, \mathbf{k}+\mathbf{G}}(\mathbf{r})=\psi_{n, \mathbf{k}}(\mathbf{r})$. Consequently, the tangential wave vectors of the air and honeycomb photonic crystals are equal in the first Brillouin zone of the reciprocal space. The wave vector of the refracted wave centered at the $\Gamma$ point has its end points with the frequency $f=0.295 \times 2 \pi C / a$ on the equal frequency contour of the honeycomb photonic crystal. The conservation of the wave vectors was taken into consideration; accordingly, there were two possible end points. The propagation direction of the refraction wave is oriented to the direction of group velocity $\mathbf{V}_{g}=\nabla_{\mathbf{k}} \omega$, which is normally in the direction of the equal frequency contour. Because the refraction wave always propagates away from the light source, only one refraction wave is considered $\mathbf{k}_{p c}$. The group velocity direction is in the propagation direction. In the infinite periods of photonic crystals it can be proven by analytic methods that the group velocity coincides with the energy velocity [4]. The direction of the group velocity was opposite to the direction of the refraction wave vector $\mathbf{V}_{g} \cdot \mathbf{k}_{p c}<0$. Furthermore, the refraction wave vector was not necessarily antiparallel to the direction of the group velocity. The refraction wave vector is the wave vector of phase velocity. The refraction wave vector of the phase velocity is antiparallel to the direction of the group velocity, as the shape of the equal frequency contour is nearly round. The phenomena observed in our experiment are similar to the left-handed behavior [6]. As long as the shape of the equal frequency contour with a certain frequency is nearly round and isotropic, in principle there is 


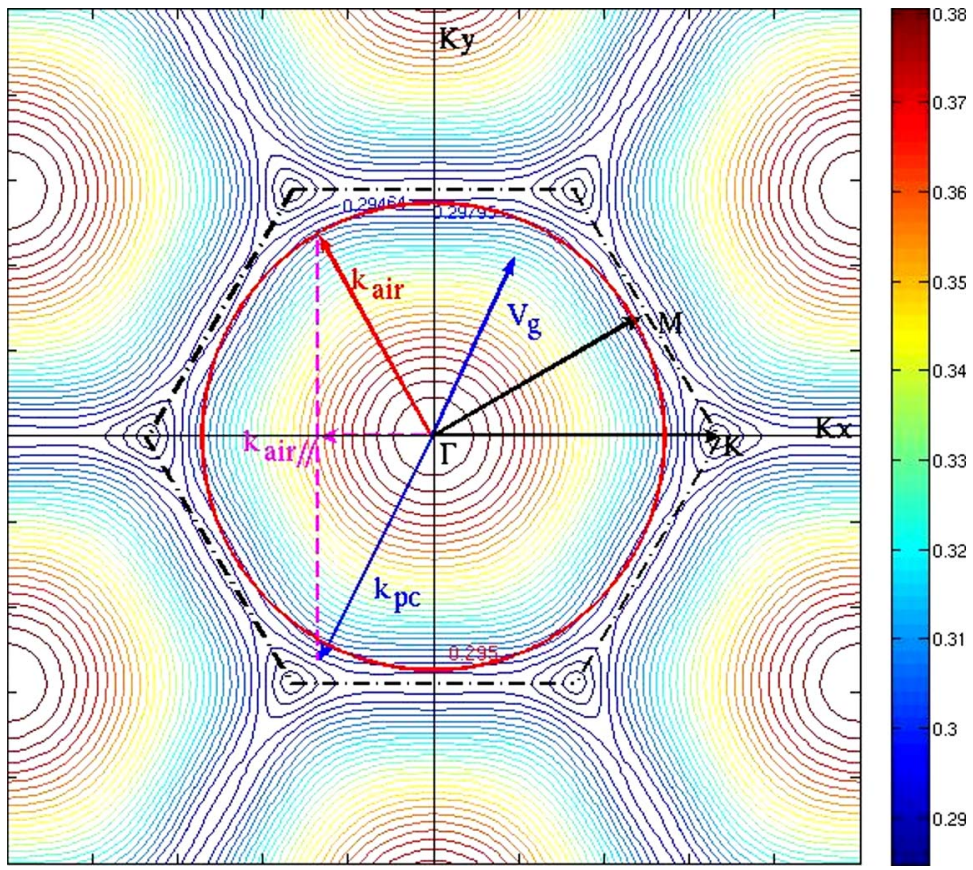

Fig. 3. (Color online) Equal frequency contours of the second band of the TM mode for the two-dimensional honeycomb photonic crystals studied in this paper. The dashed-dotted lines denote the first Brillouin boundaries. The outlined circle is the equal frequency contour of air for $f=0.295 \times 2 \pi C / a$. The arrow in the upper-left quadrant indicates the wave vector of the incident wave $\mathbf{k}_{\text {air }}$ with incident angle $30^{\circ}$ with respect to $\mathbf{k}_{y}$. The narrow blue arrow presents the refraction wave vector $\mathbf{k}_{p c}$ and the wide blue arrow denotes the direction of group velocity of the refraction wave $\mathbf{V}_{g}$.

almost no directional dependence of the incident wave for the negative refraction occurrence. Consequently, the negative refraction phenomenon occurs if a light with the TM mode is obliquely incident from the $\mathbf{k}_{y}$ direction, and if the electromagnetic wave with the $E$ polarization is obliquely incident from the $\mathbf{k}_{x}$ direction. For this purpose, electromagnetic propagation through a slab put in the air was taken into consideration and the time change of the

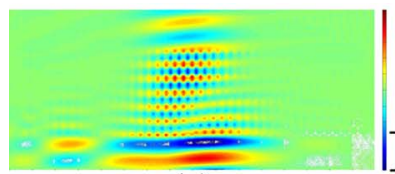

(a)

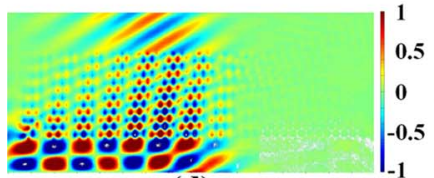

(d)

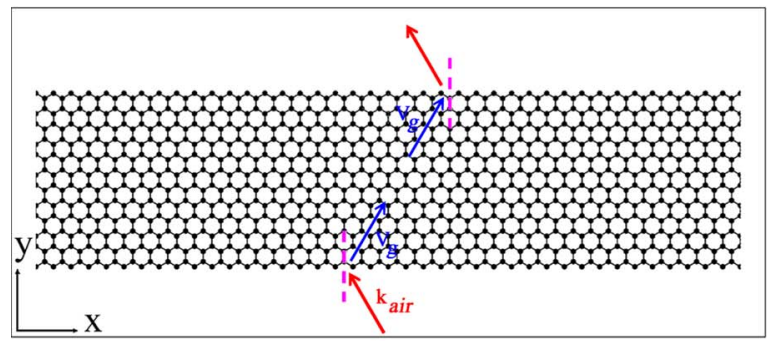

(g)

(b)

(e)
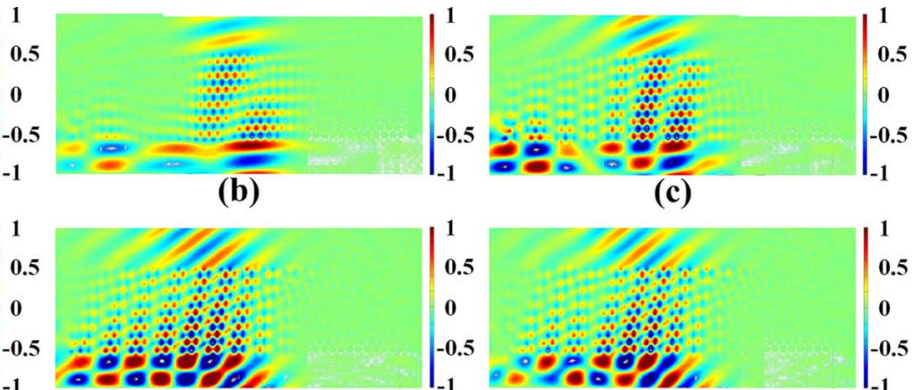

(c)

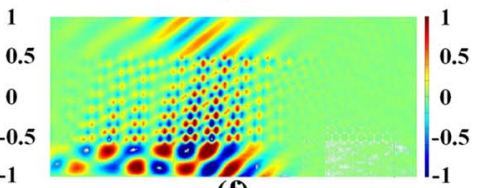

(f)

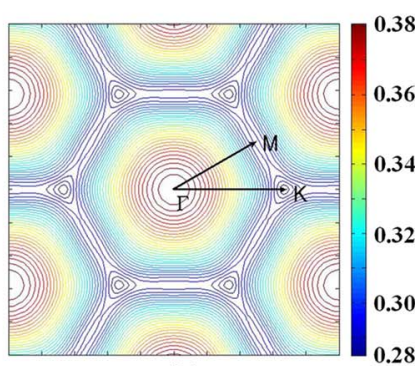

(h)

Fig. 4. (Color online) Amplitude snapshots of the electric fields cross a flat two-dimensional photonic crystal slab with the dielectric constant $\varepsilon / \varepsilon_{0}=13.0$. A plane wave with frequency $f=0.295 \times 2 \pi C / a$ is impinged on the slab with different incident angles with respect to the surface-normal: (a) $5^{\circ}$; (b) $10^{\circ}$; (c) $20^{\circ}$; (d) $30^{\circ}$; (e) $40^{\circ}$; and (f) $45^{\circ}$. (g) Photonic crystal slab. The slab is put in the vacuum and measured as $40 \times 18.7$ in terms of lattice constant $a$. For the first vacuum-honeycomb interface, the incident wave vector $\mathbf{k}_{\text {air }}$, and the group velocity of the refraction wave vector $\mathbf{V}_{g}$ are indicated by the left-pointed and right-pointed arrows, respectively. The vertical dashed lines denote the normal vector of the interface. (h) Equal frequency contours of the honeycomb photonic crystal. The $\Gamma-K$ is parallel to the $x$ direction. 
spatial distribution of the electromagnetic field was calculated using the MPI FDTD method.

Figure 4 displays a snapshot of the electric field when a collimated plane wave, imitated by the Gaussian beam, was incident at different angles on a slab of the above honeycomb photonic crystals. The length and width of the slab were 40 and 18.7, respectively, in terms of the lattice constant $a$, and the slab was set in air. The orientation of the slab is indicated in Fig. 4(g). The frequency of the incident wave is $0.295 \times 2 \pi C / a$, which is the frequency of the all-angle negative refraction denoted in Fig. 3. A total of six incident angles were examined. These incident wave vector angles cover the significant symmetric wave vectors $\Gamma-K$ and $\Gamma-M$, since the irreducible Brillouin zone of the honeycomb has sixfold symmetry. A highly reflective wave was found at most of the various angles of incidence. These results may be explained by considering the mismatch of the impedance $(\mu / \varepsilon)^{1 / 2}$ between the photonic crystal and air. In Fig. 4, the direction of the incident light was set at the right-hand side of the normal direction. The wave vector that was in the photonic crystals appeared on the same side as the illuminated wave vector. The wave vector of the outgoing light from the photonic crystal appeared on the same side as the wave vector in the photonic crystals. Regardless of the angle of incidence of the wave on the slab, the wave always tended to be negatively refracted. These results agree with the theory of the negative refraction photonic crystals $[11,12,14]$ and are similar to the results of [22]. The refraction angle of the outgoing light was highly relative to the angle of incidence of the incident light between incident angles of $5^{\circ}$ and $30^{\circ}$; whereas, the refraction direction of the outgoing light was not closely associated with incident light between $35^{\circ}$ and $45^{\circ}$. The results indicate that the equal frequency contour $f=0.295 \times 2 \pi C / a$ is not round enough to cause these phenomena between incident angles of $40^{\circ}$ and $45^{\circ}$. Outgoing light with incident angles between $5^{\circ}$ and $10^{\circ}$ had lower transmission rates than others. One possible explanation is that the incident light excites the eigenmodes of the honeycomb photonic crystal, so that as more eigenmodes are excited, more energy is transmitted, and that incident light with a larger incident angle more easily excites the eigenmodes between incident angles of $30^{\circ}$ and $40^{\circ}$.

The electromagnetic propagation through the slab of honeycomb photonic crystals with a different orientation was also investigated. The unit cell of the honeycomb photonic crystals was rotated by $90^{\circ}$ along the $z$ axis and arranged periodically along two of its axes, so the first Brillouin zone was turned to $90^{\circ}$. The flat bulk photonic crystal was $44.4 \times 18.6$ in terms of lattice constant $a$. A plane wave was launched into the slab and the normal direction of the incident light was parallel with $\Gamma-K$. All the physical parameters of the incident wave are the same as above. The results are shown in Fig. 5. No negative refractions were found at any angle of incidence, and the lights were more or less bent in the direction of $\Gamma-M$. This shows that honeycomb photonic crystals are not allangle negative refraction material in this orientation, although the equal frequency contour with frequency $f$ $=0.295 \times 2 \pi C / a$ is nearly round. These results can be explained by considering the group theory for twodimensional photonic crystals $[4,23,25]$. In Fig. 2, the second band along the $\Gamma-K$ direction for the TM mode can be labeled as an uncoupled mode called mode B. The wave function of the electric field of mode $B$ is antisymmetric under the mirror reflection at the plane that contains the $z$ axis and the connecting line of $\Gamma-K$. Once the wave

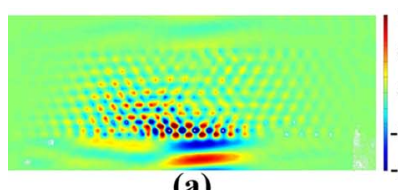

(a)

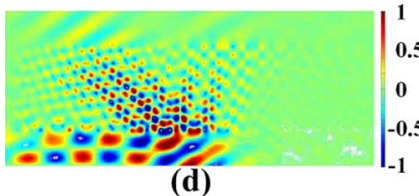

(d)

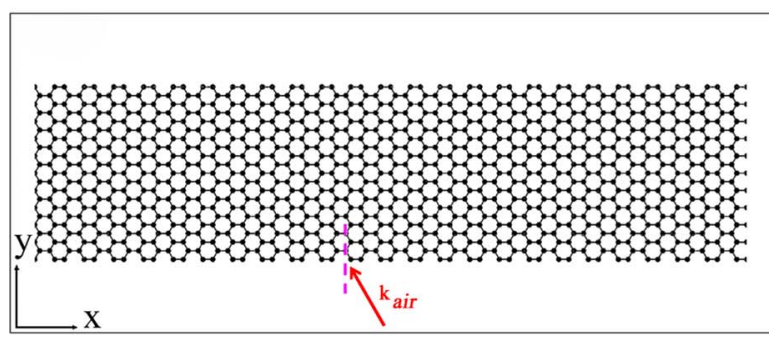

(g)

(b)

(e)
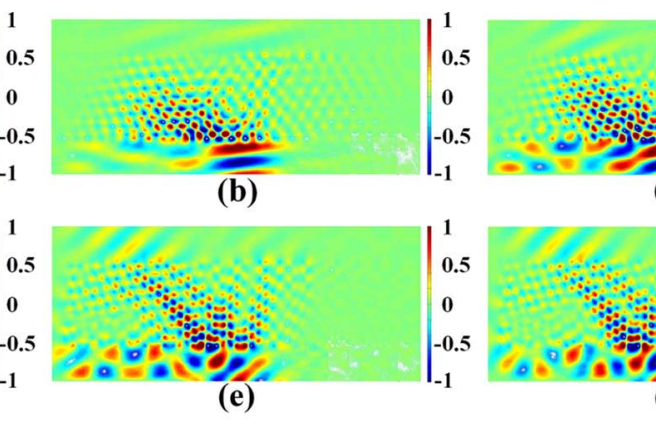

(c)
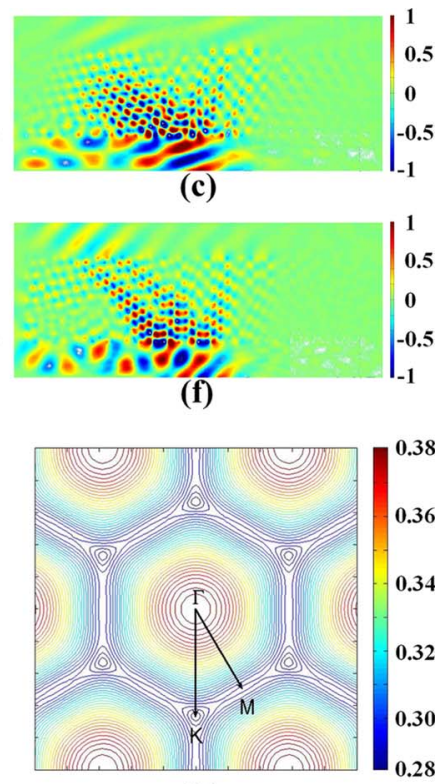

(h)

Fig. 5. (Color online) Amplitude snapshots of the electric field across a flat slab photonic crystal with the dielectric constant $\varepsilon / \varepsilon_{0}$ $=13.0$. A plane wave with a frequency of $f=0.295 \times 2 \pi C / a$ is incident on the slab with several incident angles with respect to the $y$ axis: (a) $5^{\circ}$; (b) $10^{\circ}$; (c) $20^{\circ}$; (d) $30^{\circ}$; (e) $40^{\circ}$; and (f) $45^{\circ}$. (g) Flat slab of the honeycomb photonic crystal. The slab is set in the vacuum and was $44.4 \times 18.6$ in terms of lattice constant $a$. For the first vacuum-honeycomb interface, the incident wave vector and the normal vector are indicated by the diagonal arrow and the vertical dashed line, respectively. (h) Equal frequency contours for the orientation of Fig. 5(g). The $\Gamma-K$ is antiparallel to the $y$ direction. 
function of the propagation light has mirror reflection symmetry at the plane that includes the $z$ axis and the wave vector direction $\mathbf{k}_{\text {air }}$, the mode $B$, which is in the honeycomb photonic crystals, cannot be excited by external waves at a normal angle of incidence. Even though the equal frequency contour is isotropic for a defined frequency, this result suggests that the symmetric properties of the photonic crystals cannot be ignored and that the photonic crystals of negative refraction may not be regarded as an effective medium for some special incident directions.

Figure 5(a), whose incident direction is near $\Gamma-K$, did not show negative refraction. In contrast, Fig. 4(d) shows that the wave has an incident angle of $30^{\circ}$ with respect to the normal direction, and that the incident angle is in the $\Gamma-K$ direction. Figure $4(d)$ can be explained as follows. The wave function of the incident light on the interface between the vacuum and photonic crystals begins to alternate between positive and negative amplitudes around an incident angle of $30^{\circ}$. The conservation of the tangential wave vectors is taken into consideration, so the mode $\mathrm{B}$ can be excited by an external incident field.

\section{CONCLUSIONS}

We originally assumed that perfect circle equal frequency contours of all-angle negative refraction would be sufficient to guarantee the all-angle negative refraction phenomena. Our findings, however, go against this assumption. The all-angle negative refraction phenomena that were found in the photonic crystals depend on the orientation of the photonic crystals, even if the equal frequency contour was in the shape of a perfect circle. We can conclude with certainty that mode $B$ in the photonic crystals cannot be excited by an external incident field in the nearly normal incident direction. These results are in substantial agreement with those of Sakoda [25]; conversely, these findings seem inconsistent with the definition of all-angle negative refraction; namely, that allangle negative refraction takes place for all angles. The wave function of the mode $B$ could not be excited by an external light with a normal angle of incidence. On the other hand, the wave function of mode B can be excited by an external light at an oblique angle of incidence, so the negative refraction can occur in the specific orientation of the photonic crystals. These findings provide evidence that the symmetrical properties of the photonic crystals should be considered along with the all-angle negative refraction phenomena. The negative refraction phenomena of triangular photonic crystals behave the same as those of honeycomb photonic crystals. Moreover, the approach outlined in this study can be replicated in threedimensional photonic crystals.

\section{ACKNOWLEDGMENTS}

K.-M. Lin thanks Yih-Peng Chiou, Wei-Chih Liu, DinPing Tsai, Chaur-Yi Chou, Tai-I Weng, and Tak-Pong Woo for their valuable comments during the development of the MPI FDTD program. This research was partially sponsored by the National Science Council and the National Center for Theoretical Sciences (NCTS) of Taiwan.
We also thank the National Center for High-Performance Computing of Taiwan and the Computer and Information Networking Center of National Taiwan University for providing CPU time.

\section{REFERENCES}

1. E. Yablonovitch, "Inhibited spontaneous emission in solidstate physics and electronics," Phys. Rev. Lett. 58, 2059-2062 (1987).

2. S. John, "Strong localization of photons in certain disordered dielectric superlattices," Phys. Rev. Lett. 58, 2486-2489 (1987).

3. J. D. Joannopoulos, R. D. Meade, and J. N. Winn, Photonic Crystals (Princeton U. Press, 1995).

4. K. Sakoda, Optical Properties of Photonic Crystals (Springer, 2001).

5. A. Yariv and P. Yeh, Optical Waves in Crystals-Propagation and Control of Laser Radiation (Wiley Interscience, 2003).

6. V. G. Veselago, "The electrodynamics of substances with simultaneously negative values of permittivity and permeability," Sov. Phys. Usp. 10, 509-514 (1968).

7. J. B. Pendry, "Negative refraction makes a perfect lens," Phys. Rev. Lett. 85, 3966-3969 (2000).

8. D. R. Smith, W. J. Padilla, D. C. Vier, S. C. Nemat-Nasser, and S. Schultz, "Composite medium with simultaneously negative permeability and permittivity," Phys. Rev. Lett. 84, 4184-4187 (2000).

9. R. A. Shelby, D. R. Smith, and S. Schultz, "Experimental verification of a negative index of refraction," Science 292, 77-79 (2001).

10. C. Luo, M. Ibanescu, E. J. Reed, S. G. Johnson, and J. D. Joannopoulos, "Doppler radiation emitted by an oscillating dipole moving inside a photonic band-gap crystal," Phys. Rev. Lett. 96, 043903 (2006).

11. M. Notomi, "Theory of light propagation in strongly modulated photonic crystals: Refractionlike behavior in the vicinity of the photonic band gap," Phys. Rev. B 62, 10696-10705 (2000).

12. C. Luo, S. G. Johnson, J. D. Joannopoulos, and J. B. Pendry, "All-angle negative refraction without negative effective index," Phys. Rev. B 65, 201104(R) (2000).

13. P. V. Parimi, W. T. Lu, P. Vodo, and S. Sridhar, "Photonic crystals: imaging by flat lens using negative refraction," Nature 426, 404 (2003)

14. E. Cubukcu, K. Aydin, E. Ozbay, S. Foteinopoulou, and C. M. Soukoulis, "Subwavelength resolution in a twodimensional photonic-crystal-based superlens," Phys. Rev. Lett. 91, 207401 (2003)

15. X. Wang, Z. F. Ren, and K. Kempa, "Unrestricted superlensing in a triangular two dimensional photonic crystal," Opt. Express 12, 2919-2924 (2004).

16. C.-H. Kuo and Z. Ye, "Optical transmission of photonic crystal structures formed by dielectric cylinders: evidence for non-negative refraction," Phys. Rev. E 70, 056608 (2004).

17. H.-T. Chien, H.-T. Tang, C.-H. Kuo, C.-C. Chen, and Z. Ye, "Directed diffraction without negative refraction," Phys. Rev. B 70, 113101 (2004).

18. L.-S. Chen, C.-H. Kuo, and Z. Ye, "Guiding optical flows by photonic crystal slabs made of dielectric cylinders," Phys. Rev. E 69, 066612 (2004).

19. Z.-Y. Li and L.-L. Lin, "Evaluation of lensing in photonic crystal slabs exhibiting negative refraction," Phys. Rev. B 68, 245110 (2003)

20. S. Foteinopoulou and C. M. Soukoulis, "Negative refraction and left-handed behavior in two-dimensional photonic crystals," Phys. Rev. B 67, 235107 (2003).

21. R. Moussa, S. Foteinopoulou, L. Zhang, G. Tuttle, K. Guven, E. Ozbay, and C. M. Soukoulis, "Negative refraction and superlens behavior in a two-dimensional photonic crystal," Phys. Rev. B 71, 085106 (2005).

22. R. Gajić, R. Meisels, F. Kuchar, and K. Hingerl, "All-angle 
left-handed negative refraction in Kagomé and honeycomb lattice photonic crystals," Phys. Rev. B 73, 165310 (2006).

23. W. M. Robertson, G. Arjavalingam, R. D. Meade, K. D. Brommer, A. M. Rappe, and J. D. Joannopoulos, "Measurement of photonic band structure in a twodimensional periodic dielectric array," Phys. Rev. Lett. 68, 2023-2026 (1992).

24. K. Sakoda, "Optical transmittance of a two-dimensional triangular photonic lattice," Phys. Rev. B 51, 4672-4675 (1995).

25. K. Sakoda, "Symmetry, degeneracy, and uncoupled modes in two-dimensional photonic lattices," Phys. Rev. B 52 7982-7986 (1995).

26. A. Martínez, H. Míguez, A. Griol, and J. Martí, "Experimental and theoretical analysis of the self-focusing of light by a photonic crystal lens," Phys. Rev. B 69, 165119 (2004).

27. R. L. Chern, C. C. Chang, and R. R. Hwang, "Large full band gaps for photonic crystals in two dimensions computed by an inverse method with multigrid acceleration," Phys. Rev. E 68, 026704 (2003).

28. T.-I. Weng and G. Y. Guo, "Band structure of honeycomb photonic crystal slabs," J. Appl. Phys. 99, 093102 (2006).

29. K. M. Leung and Y. F. Liu, "Full vector wave calculation of photonic band structures in face-centered-cubic dielectric media," Phys. Rev. Lett. 65, 2646-2649 (1990).

30. Z. Zhang and S. Satpathy, "Electromagnetic wave propagation in periodic structures: Bloch wave solution of Maxwell's equations," Phys. Rev. Lett. 65, 2650-2653 (1990).
31. K. M. Ho, C. T. Chan, and C. M. Soukoulis, "Existence of a photonic gap in periodic dielectric structures," Phys. Rev. Lett. 65, 3152-3155 (1990).

32. S. G. Johnson and J. D. Joannopoulos, "Block-iterative frequency-domain methods for Maxwell's equations in a planewave basis," Opt. Express 8, 173-190 (2001).

33. K. S. Yee, "Numerical solution of initial boundary value problems involving Maxwell's equations in isotropic media," IEEE Trans. Antennas Propag. 14, 302-307 (1966).

34. J.-P. Berenger, "A perfectly matched layer for the absorption of electromagnetic waves," J. Comput. Phys. 114, 185-200 (1994).

35. A. Taflove, Computational Electrodynamics-The FiniteDifference Time-Domain Method (Artech House, 2000).

36. D. M. Sullivan, Electrodynamics Simulation Using The FDTD Method (IEEE, 2000).

37. M. F. Su, D. A. Bader, I. El-Kady, and S.-Y. Lin, "A novel FDTD application featuring OpenMP-MPI hybrid parallelization," in Proceedings of the 2004 International Conference on Parallel Processing (IEEE, 2004), pp. 373-379.

38. Y. Aoyama and J. Nakano, RS/6000 SP: Practical MPI Programming (IBM Corporation, International Technical Support Organization, 1999), http:// www.redbooks.ibm.com/.

39. M. J. Quinn, Parallel Programming in $C$ with MPI and OpenMP (McGraw-Hill, 2003). 